\title{
ON DIRECT PRODUCTS OF INFINITE CYCLIC GROUPS
}

\section{R. J. NUNKE ${ }^{1}$}

1. Introduction. A direct product of infinite cyclic groups is a group $Z^{I}$ consisting of all functions $x: I \rightarrow Z$ (where $Z$ is the additive group of integers) with addition defined termwise. Suppose that $Z$ is given the discrete topology and $Z^{I}$ the corresponding Cartesian product topology. It is natural to ask about the structure of the closed subgroups of $Z^{I}$. We shall show that if $I$ is countable the closed subgroups of $Z^{I}$ are products, i.e., are isomorphic to groups of the form $Z^{J}$. If $I$ is uncountable there are closed subgroups which are not products. We shall show however that every direct summand of a product is a product and give necessary and sufficient conditions for a subproduct of a product to be a direct summand.

2. Topology. If $\left\{a^{n}\right\}$ is a sequence of elements of $Z^{I}$, then $a^{n} \rightarrow a$ in the product topology if, for each index $i$ in $I, a_{i}^{n}=a_{i}$ for almost all $n$. In particular $a^{n} \rightarrow 0$ if, for each $i, a_{i}^{n}=0$ for almost all $n$. Let $N$ be the set of natural numbers and for $i$ in $N$ let $\delta^{i}$ in $Z^{N}$ be such that $\delta_{j}^{i}=0$ for $i \neq j$ and $=1$ for $i=j$. We have

LEMMA 1. If $\left\{a^{n}\right\}$ is a sequence of elements of $Z^{I}$, then $a^{n} \rightarrow 0$ if and only if there is a homomorphism $h: Z^{N} \rightarrow Z^{I}$ such that $h\left(\delta^{n}\right)=a^{n}$ for all $n$ in N. This homomorphism, if it exists, is unique.

Proof. If $a^{n} \rightarrow 0$ we define $h$ by $h(x)_{i}=\sum_{n} x_{n} a_{i}^{n}$ for each $i$ in $I$. By the definition of convergence the sum used to define $h$ is finite on each coordinate. Moreover $h\left(\delta^{n}\right)=a^{n}$ so that the required $h$ exists.

Conversely suppose such an $h$ exists. Let $\pi_{i}: Z^{I} \rightarrow Z$ be the $i$ th coordinate projection. Then $\pi_{i} h: Z^{N} \rightarrow Z$ and, according to Specker [4], $\pi_{i} h\left(\delta^{n}\right)=0$ for almost all $n$. Since $\pi_{i} h\left(\delta^{n}\right)=a_{i}^{n}$ we have $a^{n} \rightarrow 0$.

Finally the uniqueness of $h$ follows from the result of Specker [4], that if $f: Z^{N} \rightarrow Z$ and $f\left(\delta^{n}\right)=0$ for all $n$, then $f=0$.

If $X$ and $Y$ are topological spaces, a map $f: X \rightarrow Y$ is sequentially continuous if $f\left(x^{n}\right) \rightarrow f(x)$ whenever $x^{n} \rightarrow x$. As a corollary to Lemma 1 we have

LEMMA 2. If I and $J$ are arbitrary nonempty sets, then every homomorphism $f: Z^{I} \rightarrow Z^{J}$ is sequentially continuous.

Presented to the Society, January 26,1961, under the title Direct products of copies of the integers; received by the editors January 25, 1961.

1 This work was supported by the National Science Foundation research contract NSF-G11098. 
Proof. Let $x^{n}$ be a sequence in $Z^{I}$ and $x^{n} \rightarrow x$. Then $x^{n}-x \rightarrow 0$. By Lemma 1 we have a homomorphism $h: Z^{N} \rightarrow Z^{I}$ such that $h\left(\delta^{n}\right)=x^{n}-x$. If $f: Z^{I} \rightarrow Z^{J}$ is a homomorphism we have $f h\left(\delta^{n}\right)=f\left(x^{n}\right)-f(x) \rightarrow 0$ so that $f\left(x^{n}\right) \rightarrow f(x)$ as required.

It is natural to ask at this point whether sequential continuity can be replaced by continuity in Lemma 2 . There are two approaches to this question. In both we note that we can take $Z^{J}=Z$. The first approach is to apply a theorem of Mazur [3] to strengthen sequential continuity to continuity. According to Mazur there is a cardinal $\alpha$ such that every sequentially continuous function $f: Z^{I} \rightarrow Z$ is continuous provided the cardinal $|I|$ of $I$ is less then $\alpha$.

The second approach notes first that every homomorphism of $Z^{I} \rightarrow Z$ is continuous if and only if

(1) $\operatorname{Hom}\left(Z^{I}, Z\right)$ is a free abelian group with a basis consisting of the coordinate projections.

This equivalence is easily proven by showing that (1) is equivalent to the conjunction of

(2) if $f: Z^{I} \rightarrow Z$ is a homomorphism, then $h\left(\delta^{i}\right)=0$ for all but a finite number of indices $i$; and

(3) if $f: Z^{I} \rightarrow Z$ is a homomorphism such that $h\left(\delta^{i}\right)=0$ for all indices $i$, then $f=0$.

The statement (2) is equivalent to Lemma 2. The statement (3) holds whenever $|I|$ is less than the first cardinal $\beta$ with a nonzero countably additive two-valued measure which is zero on finite sets. This result was announced by Ehrenfeucht and Los in [1]; a proof is given in Fuchs [2, pp. 169-171].

An examination of Mazur's paper shows that $\alpha \leqq \beta$; whether equality holds I do not know. In any event the second approach yields a simpler proof.

\section{Closed subgroups of products.}

THEOREM 3. Every closed subgroup of $Z^{N}$ is a product.

Proof. Let $P=Z^{N}$ and, for each $n$ in $N$, let $P_{n}$ be the subgroup of those $x$ in $P$ such that $x_{i}=0$ for $i<n$. Then $P_{1}, P_{2}, \ldots$ is a base at 0 for the topology on $P$. If $G$ is a subgroup of $P$, then $x$ is in $\bar{G}$ if and only if, for each $n$ in $N$, there is an element $u^{n}$ in $G$ such that $x_{i}=u_{i}^{n}$ for all $i<n$.

Let $A$ be any subgroup of $P$ and let $d_{n}$ be the g.c.d. of the integers $u_{n}$ where $u$ ranges over the elements of $A \cap P_{n}$. Since the g.c.d. of a nonempty set of integers is a linear combination of a finite number of them, there is an element $a^{n}$ in $A \cap P_{n}$ such that $a_{n}^{n}=d_{n}$. If $d_{n}=0$ we choose $a^{n}=0$. The elements $a^{n}$ have the following properties: 
(i) $a_{i}^{n}=0$ for $i<n$;

(ii) $a_{n}^{n}=0$ if and only if $a^{n}=0$;

(iii) $a_{n}^{n}$ divides $u_{n}$ for all $u$ in $A \cap P_{n}$.

In view of (i) $a^{n} \rightarrow 0$ so that, by Lemma 1 , there is a homomorphism $h: P \rightarrow P$ such that $h\left(\delta^{n}\right)=a^{n}$. For $x$ in $P$ set $x^{n}=x_{1} \delta^{1}+\cdots+x_{n-1} \delta^{n-1}$. Then $x^{n} \rightarrow x$ so that $h\left(x^{n}\right) \rightarrow h(x)$ by the sequential continuity of $h$. Since $h\left(x^{n}\right)=x_{1} a^{1}+\cdots+x_{n-1} a^{n-1}$ is in $A$ for all $n$, we have $h(x)$ in $\bar{A}$. Thus $h(P) \subseteq \bar{A}$.

To show the opposite inclusion let $y$ be any element of $\bar{A}$. Then there is a $y^{1}$ in $A$ such that $y_{1}^{1}=y_{1}$. According to (iii) (and $P_{1}=P$ ) we have $y_{1}=x_{1} a^{1}$. Hence $y-x_{1} a^{1}$ is in $\bar{A} \cap P_{2}$ which is the closure of $A \cap P_{2}$. Then there is a $y^{2}$ in $A \cap P_{2}$ such that $\left(y-x_{1} a^{1}\right)_{2}=y_{2}^{2}$. By (iii) $y_{2}^{2}=x_{2} a^{2}$ so that $y-x_{1} a^{1}-x_{2} a^{2}$ is in $\bar{A} \cap P_{3}$. Continuing we get a sequence $x=\left(x_{1}, x_{2}, \cdots\right)$ such that

$$
y-x_{1} a^{1}-x_{2} a^{2}-\cdots-x_{n-1} a^{n-1} \in \bar{A} \cap P_{n}
$$

for each $n$ in $N$. Therefore $h\left(x^{n}\right)=x_{1} a^{1}+\cdots+x_{n-1} a^{n-1} \rightarrow y$ so that $h(x)=y$ by Lemma 2. Since $y$ was arbitrary in $\bar{A}$ we have $h(P)=\bar{A}$.

For $\tau \subseteq N$ let $P(\tau)=\left\{x \mid x_{n}=0\right.$ for $\left.n \in \tau\right\}$. We define $\sigma=\left\{n \mid a^{n}=0\right\}$. Then $P=P(\sigma) \oplus P(N-\sigma)$ and each of $P(\sigma)$ and $P(N-\sigma)$ is a product. We shall show that the kernel of $h$ is $P(N-\sigma)$. Then $\bar{A}$ is isomorphic to $P(\sigma)$ which proves the theorem. It is clear from the definition of $h$ that $h(P(N-\sigma))=0$. Suppose that $x \neq 0$ is in $P(\sigma)$ and let $k$ be the first index such that $x_{k}=0$. We have $k$ in $N-\sigma$ so that $a_{k}^{k} \neq 0$. Also $h(x)_{k}=\sum_{i} x_{i} a_{k}^{i}$ with $x_{i}=0$ for $i<k$ and $a_{k}^{i}=0$ for $i>k$ (by (i)). Hence $h(x)_{k}=x_{k} a_{k}^{k} \neq 0$ because $x_{k} \neq 0$ and $a_{k}^{\boldsymbol{k}} \neq 0$. Therefore $x \neq 0$ in $P(\sigma)$ implies $h(x) \neq 0$. For an arbitrary $x$ in $P$ we have $x=x^{\sigma}+\left(x-x^{\sigma}\right)$ with $x^{\sigma}$ in $P(N-\sigma)$ and $x-x^{\sigma}$ in $P(\sigma)$. If $h(x)=0$ we have $0=h\left(x-x^{\sigma}\right)$ so that $x-x^{\sigma}=0$. Thus $h(x)=0$ implies $x=x^{\sigma}$ is in $P(N-\sigma)$ showing that $P(N-\sigma)$ is the kernel of $h$.

A slight modification of this proof gives a little more. Let $S$ be the subgroup of $P=Z^{N}$ generated by the $\delta^{n}$. Thus $S$ is the free abelian group on countably many generators. We have

THEOREM 4. Every subgroup of infinite rank of $P$ is isomorphic to a subgroup of $P$ containing $S$.

Proof. If the subgroup is $A$ we proceed as above obtaining $h$ and $\sigma$. If $N-\sigma$ is finite, then $P(\sigma)$ is finitely generated. Since $h$ maps $P(\sigma)$ isomorphically onto $\bar{A}, N-\sigma$ must be infinite if $A$ has infinite rank. For each $i$ in $N$ let $k(i)$ be the $i$ th element in order of magnitude in $N-\sigma$ and define $f: P \rightarrow P$ so that $f\left(\delta^{i}\right)=\delta^{k(i)}$. Thus $f(P)=P(\sigma)$ so that 
hf maps $P$ isomorphically onto $\bar{A}$, and $h f\left(\delta^{i}\right)=a^{k(i)}$. Then $A$ is isomorphic to $(h f)^{-1}(A)$ which contains $S$.

As noted in the introduction Theorem 3 does not generalize to products with uncountably many factors. Since each endomorphism of $P$ is continuous every direct summand of $P$ is closed and thus a product. This result does generalize as we now show.

4. Direct summands of products. For an abelian group $A$, the dual $A^{*}$ of $A$ is defined by $A^{*}=\operatorname{Hom}(A, Z)$. If $B$ is a subgroup of $A$, the annihilator $B^{\prime}$ of $B$ in $A^{*}$ is the group of all $h$ in $A^{*}$ such that $h(B)=0$. The exact sequence $0 \rightarrow B \rightarrow A \rightarrow A / B \rightarrow 0$ has a dual exact sequence $0 \rightarrow(A / B)^{*} \rightarrow A^{*} \rightarrow B^{*}$. The image of $(A / B)^{*}$ in $A^{*}$ is $B^{\prime}$. If $A=B \oplus C$ so that the first sequence above splits, then so does the second and we have $A^{*}=B^{\prime} \oplus C^{\prime}$ with $B^{\prime}$ isomorphic to $C^{*}$.

Suppose that $G=Z^{I}$ is such that $G^{*}$ is a free abelian group with a basis consisting of the coordinate projections $\pi_{i}: Z^{I} \rightarrow Z$. This will be true if $|I|$ is less than the first cardinal of nonzero measure. There is a homomorphism $\sigma: G \rightarrow G^{* *}$ defined by $\sigma(x) h=h(x)$ for $x$ in $G$ and $h$ in $G^{*}$. Under the above hypotheses $\sigma$ is an isomorphism. If $\sigma(x)=0$ we have $\pi_{i}(x)=0$ for all $i$, hence $x=0$. Thus $\sigma$ is a monomorphism. If $\mu$ is in $G^{* *}$ let $x$ in $G$ be defined by $x_{i}=\pi_{i}(x)=u\left(\pi_{i}\right)$. Then $\sigma(x) \pi_{i}$ $=u\left(\pi_{i}\right)$ for all $i$. Since the $\pi_{i}$ generate $G^{*}$ we get $\sigma(x)=a$. Thus $\sigma$ is also an epimorphism. We shall identify $G$ with $G^{* *}$ by $\sigma$.

We shall call an abelian group $A$ an $e$. $h$. group if, for each $a \neq 0$ in $A$, there is an $h$ in $A^{*}$ with $h(a) \neq 0$. The following properties of e.h. groups are either well known or easy to prove.

(1) $A$ is an e.h. group if and only if it can be embedded in some $Z^{I}$.

(2) Every subgroup of an e.h. group is one.

(3) For each $A, A^{*}$ is an e.h. group.

(4) A countable e.h. group is free.

This last property follows from (1) and Theorem 47.1 of [2].

Suppose $A$ is a subgroup of $G=Z^{I}$. Then $A^{\prime \prime}$ (the annihilator of $A^{\prime}$ in $G$ ) consists of all $x$ in $G$ such that $h(x)=0$ for all $h$ in $G^{*}$ such that $h(A)=0$. We always have $A \subseteq A^{\prime \prime}$. In view of the first paragraph of this section $A=A^{\prime \prime}$ if and only if $G / A$ is an e.h. group.

After these preliminaries we can state

TheOREM 5. Let I be a set with $|I|<\beta$ (see \$2). Then

(a) If $A$ is a direct summand of $Z^{I}$, then $A$ is a product.

(b) If $A \subseteq Z^{I}, Z^{I} / A$ is an e.h. group, and $A^{*}$ is free, then $A$ is a direct summand of $Z^{I}$.

(c) If $A \subseteq Z^{I}, Z^{I} / A$ is an e.h. group, and either $A$ is a product or $I$ is countable, then $A$ is a direct summand of $Z^{I}$. 
Proof. To prove (a) suppose that $G=Z^{I}=A \oplus B$. Then $G^{*}$ $=A^{\prime} \oplus B^{\prime}$. Since $G^{*}$ is free so are $A^{\prime}$ and $B^{\prime}$. Dualizing again we get $G=A^{\prime \prime} \oplus B^{\prime \prime}$. Since $G / A$ is an e.h. group $A^{\prime \prime}=A$. Thus $A$ is isomorphic to $B^{\prime *}$. Since $B^{\prime}$ is free $A$ is therefore a product.

In all of this proof the hypothesis that $G / A$ is an e.h. group is used to show that $A=A^{\prime \prime}$. We have an exact sequence

$$
0 \rightarrow(G / A)^{*} \rightarrow G^{*} \rightarrow A^{*} \text {. }
$$

If $A^{*}$ is free then the image of $G^{*}$ in $A^{*}$ is free so that $A^{\prime}$ (the image of $(G / A)^{*}$ in $\left.G^{*}\right)$ is a direct summand of $G^{*}$. Thus $G^{*}=A^{\prime} \oplus C$ so that $G=A^{\prime \prime} \oplus C^{\prime}$. But $A=A^{\prime \prime}$ so that $A$ is a direct summand of $G$. This proves part (b).

To prove (c) we must show that if either $A$ is a product or $I$ is countable then the image of $G^{*}$ in $A^{*}$ is free. The rest of the proof follows that of (b). Let us assume first that $I$ is countable. Then $G^{*}$ is the free abelian group on countably many generators, hence countable. The image of $G^{*}$ in $A^{*}$ is then a countable e.h. group by (3), hence free by (4). With the other hypothesis $A \approx Z^{J}$ and we want $|J|<\beta$. Now the $\operatorname{rank} r(G)$ of $G$ is $2^{|I|}$ and $r(A)=2^{|J|}$. Then $|J|$ $<2^{|J|} \leqq 2^{|I|}$. Ulam has shown in [5] that $|I|<\beta$ implies $2^{|I|}<\beta$ so we have $|J|<\beta$ as required.

Theorem 5 has two immediate corollaries.

Corollary 6. If $|I|<\beta$ and if $h: Z^{I} \rightarrow Z^{J}$, then the kernel of $h$ is a direct summand of $Z^{I}$ if and only if it is a product. The image of $h$ will then be a product also.

CoROllaRY 7. If $h$ is a homomorphism of $Z^{N}$ into an e.h. group then both the kernel and the image of $h$ are products and the kernel of $h$ is a direct summand of $Z^{N}$.

In Corollary 7 the fact that $N$ is countable is important. If $I$ is uncountable there may be subgroups $A$ of $Z^{I}$ such that $Z^{I} / A$ is an e.h. group without $A$ being a product. We can however show that $A$ must be the dual of some group.

Theorem 8. (a) For any abelian group $B, B^{*}$ can be embedded in a product $Z^{I}$ so that $Z^{I} / B$ is an e.h. group.

(b) If $|I|<\beta$ and $A$ is a subgroup of $Z^{I}$ such that $Z^{I} / A$ is an e.h. group then $A$ is isomorphic to the dual of a group $B$.

Proof. We clearly have $B^{*} \subseteq Z^{B}$ so that to prove (a) we need only show that $Z^{B} / B^{*}$ is an e.h. group. Suppose $x$ is in $Z^{B}$ but not in $B^{*}$. Then there are elements $b, c$ in $B$ such that $x(b+c)-x(b)-x(c) \neq 0$. 
If $\phi: Z^{B} \rightarrow Z$ is defined by $\phi(y)=y(b+c)-y(b)-y(c)$ for $y$ in $Z^{B}$, then $\phi\left(B^{*}\right)=0$ and $\phi(x) \neq 0$. In view of the paragraph preceding Theorem 5 this shows that $Z^{B} / B^{*}$ is an e.h. group.

Now let us assume the hypotheses of (b). Then $A^{\prime \prime}=A$. Then we have setting $G=Z^{I}$ an exact sequence

$$
0 \rightarrow(G / A)^{*} \rightarrow G^{*} \rightarrow A^{*}
$$

Let $B$ be the image of $G^{*}$ in $A^{*}$. Dualizing again we have the exact sequence

$$
0 \rightarrow B^{*} \rightarrow G \rightarrow(G / A)^{* *}
$$

where we have identified $G^{* *}$ with $G$. Now the image of $B^{*}$ in $G$ is the annihilator of the image of $(G / A)^{*}$ in $G^{*}$, i.e., $A^{\prime \prime}=A$. Therefore $A$ is isomorphic to $B^{*}$.

We should note that $B^{*}$ is a closed subgroup of $Z^{B}$. If we take $P=Z^{N}$, then $P^{*}$ is the free group on countably many generators. Thus $P^{*}$ is a closed subgroup of $Z^{P}$ such that $Z^{P} / P^{*}$ is an e.h. group. This example shows that the countability restrictions are needed in Theorem 3 and in Corollary 7. The author wishes to thank H. H. Corson for suggesting that Mazur's theorem might be used to prove the theorem of Ehrenfeucht and Los and for raising the question of the closed subgroups of $Z^{N}$.

\section{BIBLIOGRAPHY}

1. A. Ehrenfeucht and J. Eos, Sur les produits cartesiens des groupes cycliques infinis, Bull. Acad. Polon. Sci. Cl. III. 2 (1954) 261-263.

2. L. Fuchs, Abelian groups, Publishing House of the Hungarian Academy of Sciences, Budapest, 1958.

3. S. Mazur, On continuous mappings on Cartesian products, Fund. Math. 39 (1952), 229-238.

4. E. Specker, Additive Gruppen von Folgen ganzer Zahlen, Portugaliae Math. 9 (1950), 131-140.

5. S. Ulam, Zur Masstheorie in der allgemeinen Mengenlehre, Fund. Math. 16 (1930), 140-150.

UNIVERSITY OF WASHINGTON 